\title{
Improved molecular identification of Thermoactinomyces spp. associated with mushroom worker's lung by 16S rDNA sequence typing
}

\author{
JIRU XU, J.R. RAO*, B. CHERIE MILLAR, J. STUART ELBORN†‡, JAMES EVANS\$, \\ JOHN G. BARR§ and JOHN E. MOORE
}

Northern Ireland Public Health Laboratory, Department of Bacteriology, Belfast City Hospital, Belfast BT9 7AD, *Applied Plant Science Division, Department of Agriculture and Rural Development, Newforge Lane, Belfast BT9 5PX, †Department of Respiratory Medicine, Belfast City Hospital, Belfast BT9 7AD, †Department of Respiratory Medicine, Queen's University of Belfast, School of Medicine, Royal Victoria Hospital, Grosvenor Road, Belfast BT12 6BA and §Regional Mycology Reference Laboratory, Department of Microbiology, The Royal Group of Hospitals, Kelvin Building, Royal Victoria Hospital, Grosvenor Road, Belfast BT12 6BA

\begin{abstract}
Mushroom worker's lung (MWL) is a hypersensitivity pneumonitis or allergic alveolitis caused by a type III IgG-mediated immunopathogenic inflammatory reaction in the host due to the inhalation of several thermophilic organisms, including Thermoactinomyces spp. It is difficult to distinguish phenotypically the eight species of this genus; therefore, this study sought to develop an improved molecular means of identifying Thermoactinomyces spp. associated with MWL by partial 16S rDNA PCR amplification and direct sequencing. Hypervariable regions within the 16S rRNA gene, which could be employed as signature sequences of the eight individual species, were identified and employed with highly conserved flanking primers to allow initial PCR amplification, before direct DNA sequencing of the 16S rDNA amplicons. A novel 24-mer 16S rDNA oligonucleotide upstream primer was designed from in silico alignments of all Thermoactinomyces spp. and was employed in combination with downstream (reverse) 16S rDNA primers. This permitted the successful identification of all four isolates associated with mushroom workers' lung. The method may be useful in the identification of Thermoactinomyces spp. associated with allergic alveolitis or pneumonitis associated with occupational exposure in agricultural and horticultural environments.
\end{abstract}

\section{Introduction}

Mushroom worker's lung (MWL) is a hypersensitivity pneumonitis or allergic alveolitis caused by a type III IgG-mediated immunopathogenic inflammatory reaction in the host due to the inhalation of several thermophilic organisms, including members of the genera Thermoactinomyces, Saccharomonospora and Micropolyspora faeni [1]. Of these, Thermoactinomyces spp., including $T$. vulgaris, have been associated frequently with the disease $[2,3]$. The condition, as the name suggests, is prevalent in workers involved in

Received 20 June 2002; accepted 12 Aug. 2002.

Corresponding author: Dr J. E. Moore (e-mail: jemoore@, niphl.dnet.co.uk). the mushroom industry, particularly in those whose occupation involves working with phase II compost or in the spawning function of mushroom production, because of exposure to high concentrations of aerial mycelia and endospores.

Laboratory culture of causal agents of MWL may be performed from clinical specimens, including sputum and broncho-alveolar lavage (BAL) fluid, as well as from the occupational areas with greatest mycelial and spore loading, by selective culture at elevated temperature $\left(56^{\circ} \mathrm{C}\right.$ for $4-5$ days), although serological detection is most commonly employed. However, reliable identification and, in particular, speciation within the Thermoactinomyces genus, may be troublesome because of the lack of well-defined phenotypic assays to 
differentiate between the eight species currently described as belonging to this genus [4]. Furthermore, correct identification is important for epidemiological reporting, as well as for identifying strain types that may be employed for subsequent antigen extraction for serological precipitin testing in routine diagnostic laboratories.

Therefore, the aim of this study was to examine partial 16S rDNA PCR amplification and direct sequencing as an improved molecular means of identification of Thermoactinomyces spp. associated with MWL.

\section{Materials and methods}

\section{PCR primer selection}

The complete 16S rDNA sequences of the eight species of Thermoactinomyces [4], including the following organisms: T. candidus (GenBank accession no. AF138732), T. dichotomicus (AF138733), T. intermedius (AF138734), T. peptonophilus (AF138735), T. putidus (AF138736), T. sacchari (AF138737), T. thalpophilus (AF138738) and T. vulgaris (AF138739), were aligned by employing the Clustal method software (DNAstar, Wisconsin, USA) as shown in Fig. 1. Universal 16S rDNA PCR primers were selected to span at least two-thirds of the 16S rDNA gene, to include most regions of hypervariability between the species lying between position 314 and 1370, and included the reverse primers PSR [5] and P13P [6], as described in relation to T. vulgaris (AF138739) (Fig. 2). A novel forward primer, $\mathrm{XB} 1$, with the sequence $5^{\prime}$ CAG ACT CCT ACG GGA GGC AGC AGT-3' was designed from the complete 16S rDNA alignment of the eight species and was selected at position 314-337 (T. vulgaris AF138739) which represented 100\% sequence homology among all eight species (Fig. 2).

\section{Extraction and PCR amplification of microbial DNA}

Four unidentified isolates associated with MWL were examined. All were purified on Colombia agar base (CM331, Oxoid) supplemented with defibrinated horse blood (Oxoid) $5 \% \mathrm{v} / \mathrm{v}$ and D-glucose $1 \% \mathrm{w} / \mathrm{v}$ and were incubated at $56^{\circ} \mathrm{C}$ for $4-5$ days. Genomic DNA was extracted from a single colony with the Roche High Purity PCR Template kit (Roche Diagnostics), in accordance with the manufacturer's instructions. All reaction mixes were set up in a PCR hood in a room separate from that used to extract DNA and the amplification and post-PCR room to minimise contamination, in accordance with the laboratory guidelines of Millar et al. [7]. PCR reaction mixes $(50 \mu \mathrm{l})$ contained: $10 \mathrm{mM}$ Tris-HCl, $\mathrm{pH} 8.3,50 \mathrm{mM} \mathrm{KCl}$, $2.5 \mathrm{mM} \mathrm{MgCl} 2,200 \mu \mathrm{M}$ (each) dATP, dCTP, dGTP and dTTP, $1.25 \mathrm{U}$ of Taq DNA polymerase (Amplitaq; Perkin Elmer), $0.2 \mu \mathrm{M}$ (each) of the 16S rRNA primers, as detailed (Fig. 2) and $4 \mu \mathrm{l}$ of DNA template containing c. $50 \mathrm{ng}$ of DNA/ml of extract. Following a 'hot start', the reaction mixtures were subjected to the following thermal cycling conditions in a Perkin Elmer 2400 thermocycler: $96^{\circ} \mathrm{C}$ for $3 \mathrm{~min}$ followed by 40 cycles of $96^{\circ} \mathrm{C}$ for $1 \mathrm{~min}, 55^{\circ} \mathrm{C}$ for $1 \mathrm{~min}, 72^{\circ} \mathrm{C}$ for $1 \mathrm{~min}$, followed by a final extension at $72^{\circ} \mathrm{C}$ for $10 \mathrm{~min}$. During each run, molecular grade water (Biowhittaker) instead of DNA was included randomly as a negative control and Staphylococcus aureus DNA was included as a positive control. After amplification, portions $(15 \mu \mathrm{l})$ were removed, electrophoresed $(80 \mathrm{~V}, 45 \mathrm{~min})$ in agarose (Gibco) gels $2 \% \mathrm{w} / \mathrm{v}$ in TAE buffer $(40 \mathrm{mM}$ Tris, $20 \mathrm{mM}$ acetic acid, $1 \mathrm{mM}$ EDTA, $\mathrm{pH} 8.3$ ) and stained with ethidium bromide $(5 \mu \mathrm{g} / 100 \mathrm{ml})$. Gels were visualised under UV illumination with a gel image analysis system (UVP Products) and all images were archived as digital $\left(^{*}\right.$.bmp) graphic files.

\section{Sequencing of amplicons and analysis of sequence data}

Amplicons for sequencing were purified with the QIAquick PCR purification kit (Qiagen) and eluted in Tris-HCl (10 mM, pH 8.5) before sequencing. Cy-5' labelled primer, XB1, was prepared and used for sequencing in the forward direction with the ALF Express II (Amersham-Pharmacia, Bucks) employing the Thermo Sequenase fluorescent-labelled primer cycle sequencing kit with 7-deaza-dGTP (Amersham Pharmacia Biotech; no. RPN 2438) $\left(96^{\circ} \mathrm{C}\right.$ for $1 \mathrm{~min}$, followed by 25 cycles of $96^{\circ} \mathrm{C}$ for $10 \mathrm{~s}, 50^{\circ} \mathrm{C}$ for $5 \mathrm{~s}$, $60^{\circ} \mathrm{C}$ for $5 \mathrm{~s}$, followed by a $4^{\circ} \mathrm{C}$ hold). The sequences obtained were compared with those stored in the GenBank data system with BLAST alignment software (http://www.blast.genome.ad.jp/). Sequence homology identity was determined in accordance with the criteria described previously [8].

\section{Results and discussion}

All four unknown thermophilic isolates examined generated PCR products of the expected size of $c$. $762 \mathrm{bp}$ and $1056 \mathrm{bp}$ for primer combinations XB1/PSR and XB1/P13P, respectively (Fig. 3). Subsequent sequence analysis of the 1056-bp amplicon identified the isolates as shown in Table 1 and the sequences have been deposited in GenBank, as detailed (Table 1).

In this study, the initial objective was to identify a highly conserved upstream region of the 16S rRNA gene in Thermoactinomyces spp. that could act as the forward primer in a PCR reaction, in combination with a highly conserved downstream $16 \mathrm{~S}$ rDNA primer. The approach adopted was to identify hypervariable regions within the 16S rRNA gene, which could be employed as signature sequences of the eight individual species within this genus and to employ highly conserved flanking primers to allow initial PCR amplification, 


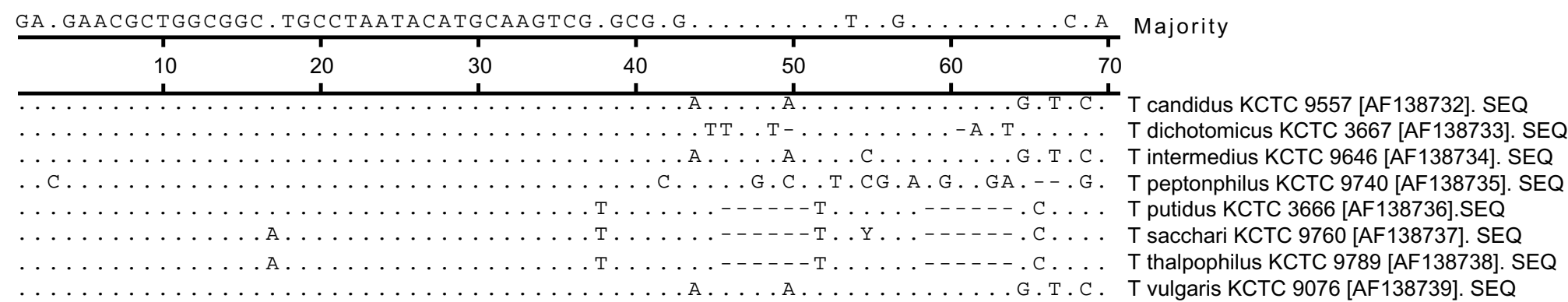

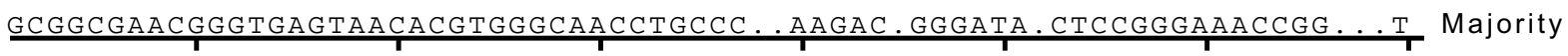 \\ 80}

100

110

120

130

140

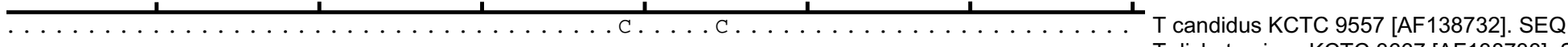

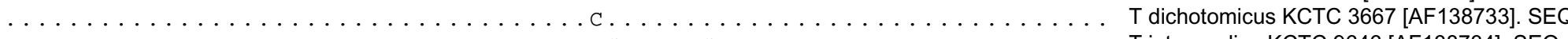

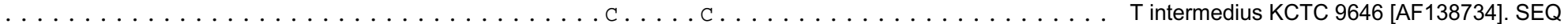

$\ldots \ldots \ldots \ldots \ldots \ldots \ldots \ldots \ldots \ldots \ldots \ldots \ldots \ldots \ldots \ldots \ldots \ldots \ldots$. . . . . . . . . . . peptonphilus KCTC 9740 [AF138735]. SEQ

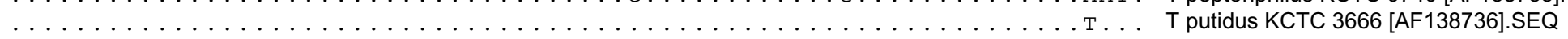

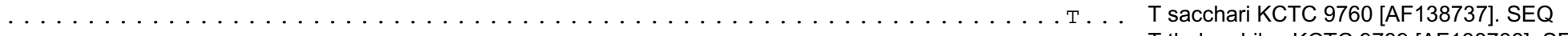

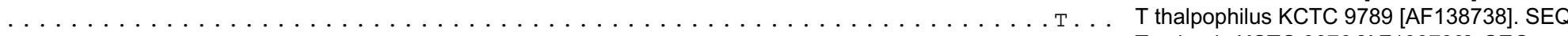

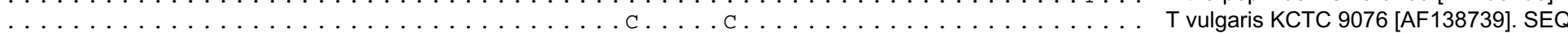

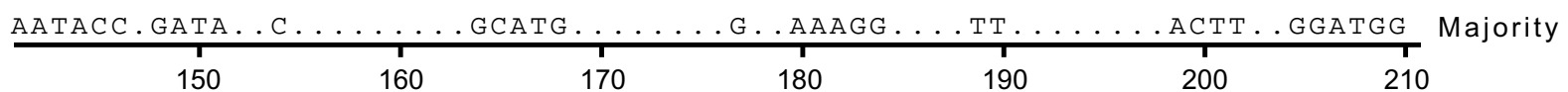

139

141

139

129

129

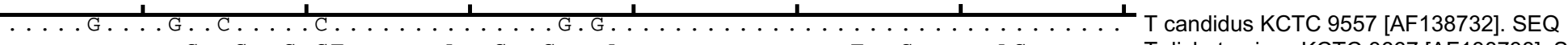

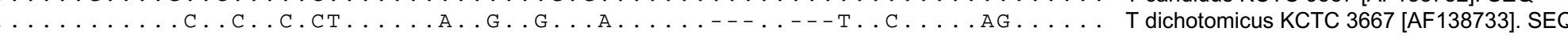

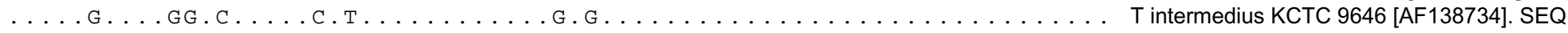

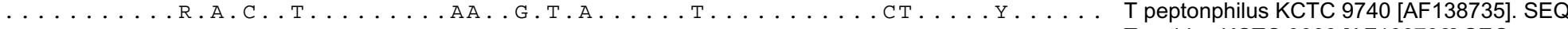
$\ldots \ldots \ldots \ldots$. . . . . . . . . . . . . . . . . .-. . . . . . . . . . . T putidus KCTC 3666 [AF138736].SEQ $\ldots \ldots \ldots$. . . . . . . . . . . . . . . . . . . . . . . . . . . . . T sacchari KCTC 9760 [AF138737]. SEQ $\ldots \ldots \ldots \ldots$. $\ldots \ldots \ldots \ldots$. . . . . A . . .-. . . .-. . . . . . . T thalpophilus KCTC 9789 [AF138738]. SEQ

GCCCGCGGCGCATTAGCT.GT.GGTGAGGTAA.GGCTCACC.AGGC.ACGATGCGTAGCCGACCTGAgAG Majority

\begin{tabular}{|c|c|c|c|c|c|c|c|c|}
\hline & 220 & 230 & 240 & 250 & 260 & 270 & 280 & \\
\hline 211 & & & & & & & & T candidus KCTC 9557 [AF138732]. SEQ \\
\hline 203 & • & 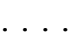 & & • & & 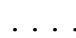 & .. & T dichotomicus KCTC 3667 [AF138733]. SEQ \\
\hline 211 & & . G. & & & & & & T intermedius KCTC 9646 [AF138734]. SEQ \\
\hline 209 & & $\cdots$ & & & & & & T peptonphilus KCTC 9740 [AF138735]. SEQ \\
\hline 193 & . & . T. & & $\cdot$ & & & & T putidus KCTC 3666 [AF138736].SEQ \\
\hline 193 & 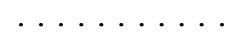 & & & & & & & T sacchari KCTC 9760 [AF138737]. SEQ \\
\hline 193 & & & & & & & & T thalpophilus KCTC 9789 [AF138738]. SEQ \\
\hline 211 & & G & & & & & & T vulgaris KCTC 9076 [AF138739]. SEQ \\
\hline
\end{tabular}

Fig. 1. Nucleotide alignments of part of the 16S rRNA gene of eight species of Thermoactinomyces. The consensus sequence is shown along the title bar and only differences are displayed. 
GGTGA. CGGCCACACTGGGACTGAGACACGGCCCAGACTCCTACGGGAGGCAGCAGTAGGGAATTTCCG

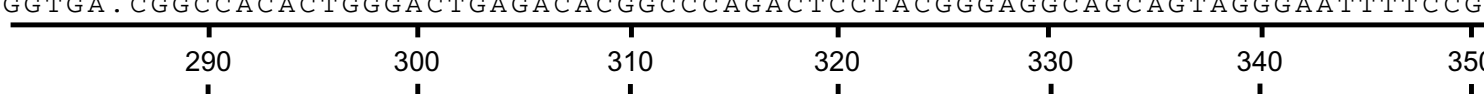

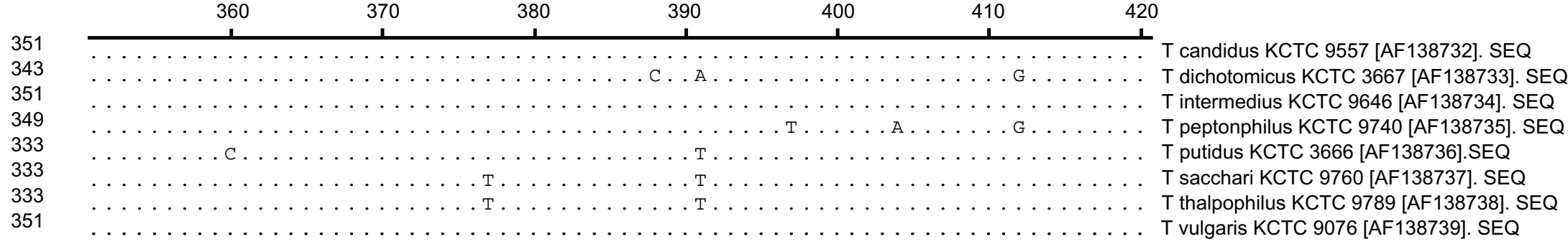

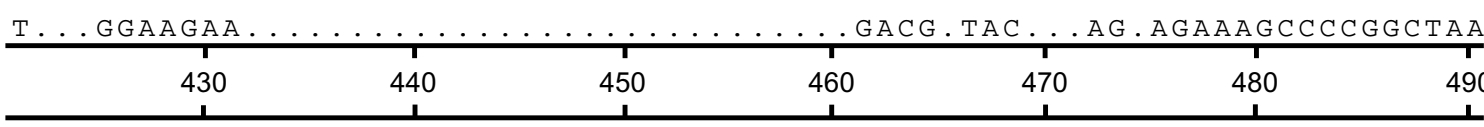

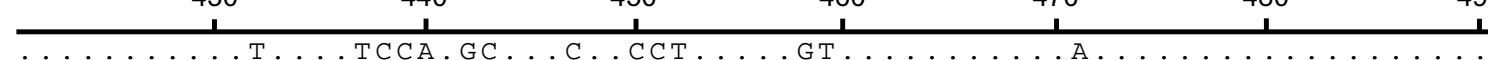

$\ldots G \ldots \ldots \ldots-1, \ldots \ldots \ldots$. $\ldots \ldots \ldots \ldots \ldots$ $\ldots \ldots \ldots$ T. . . GA. ..... С . . . GA. . . . . T .-. GA...... . . Majority T candidus KCTC 9557 [AF138732]. SEQ T dichotomicus KCTC 3667 [AF138733]. SEQ T intermedius KCTC 9646 [AF138734]. SEQ T peptonphilus KCTC 9740 [AF138735]. SEQ T putidus KCTC 3666 [AF138736].SEQ T sacchari KCTC 9760 [AF138737]. SEQ

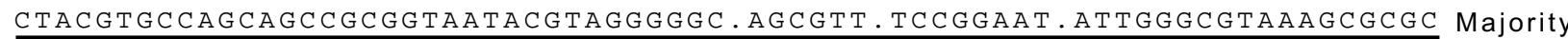

\begin{tabular}{|c|c|c|c|c|c|c|c|}
\hline 500 & 510 & 520 & 530 & 540 & 550 & $\overline{560}$ & \\
\hline & & & & & & & T candidus KCTC 9557 [AF138732]. SEQ \\
\hline$\because$ & & & & & & . & T dichotomicus KCTC 3667 [AF138733]. SEQ \\
\hline 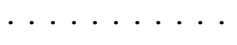 & $\cdots$ & $\cdots$ & . & . & $\cdots$ & . & T intermedius KCTC 9646 [AF138734]. SEQ \\
\hline$\cdots$ & $\cdots$ & & .. & . & & . & T peptonphilus KCTC 9740 [AF138735]. SEQ \\
\hline$\cdots$ & 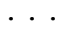 & & & & & . & T putidus KCTC 3666 [AF138736].SEQ \\
\hline$\cdot$ & 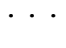 & & & & & . & T sacchari KCTC 9760 [AF138737]. SEQ \\
\hline & & & & & & & $\begin{array}{l}\text { T thalpophilus KCTC } 9789 \text { [AF138738]. SEQ } \\
\text { T vulgaris KCTC } 9076 \text { [AF138739]. SEQ }\end{array}$ \\
\hline
\end{tabular}

Fig. 1. (continued) 


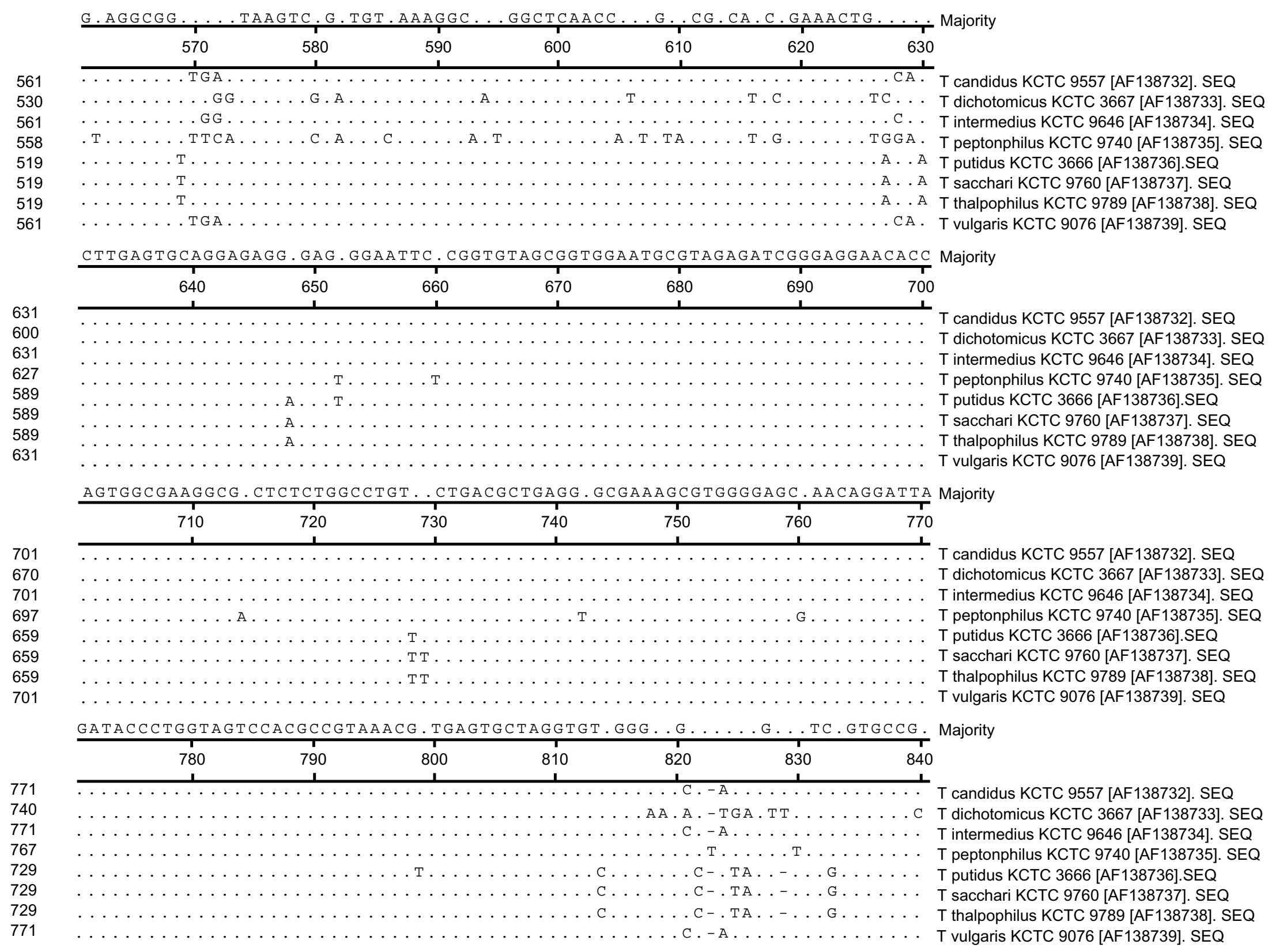

Fig. 1. (continued) 


910
879
910
907
867
867
867
910

\begin{tabular}{|c|c|c|c|c|c|c|}
\hline 920 & 930 & 940 & 950 & 960 & 970 & 980 \\
\hline
\end{tabular}

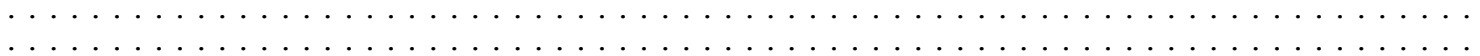

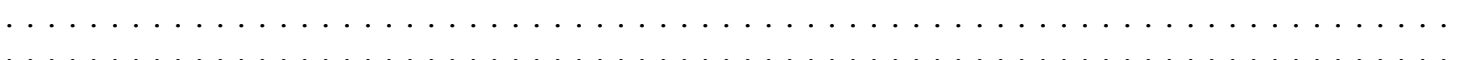

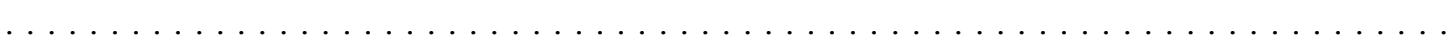

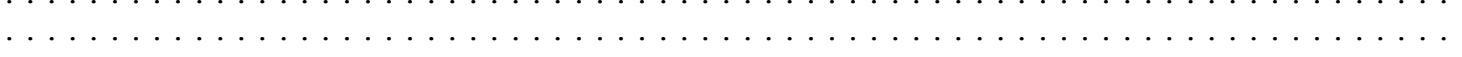

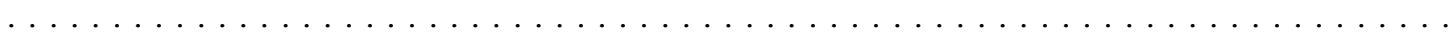

T candidus KCTC 9557 [AF138732]. SEQ T dichotomicus KCTC 3667 [AF138733]. SEQ T intermedius KCTC 9646 [AF138734]. SEQ T peptonphilus KCTC 9740 [AF138735]. SEQ T putidus KCTC 3666 [AF138736].SEQ

T sacchari KCTC 9760 [AF138737]. SEQ

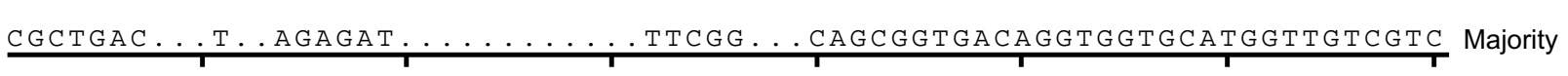

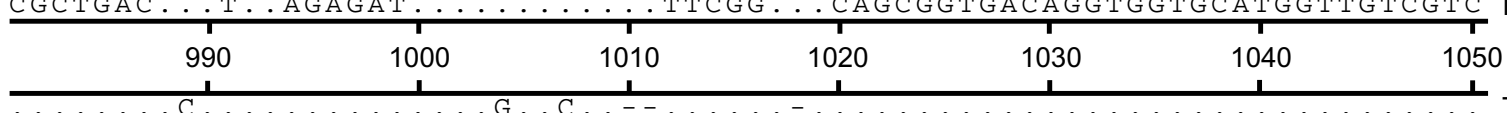

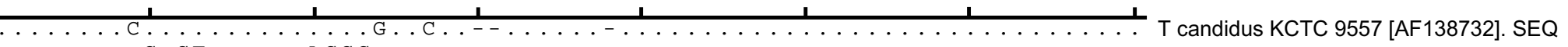

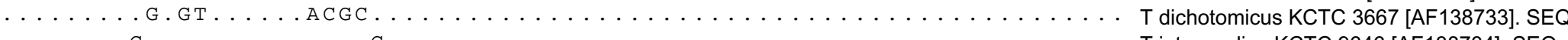

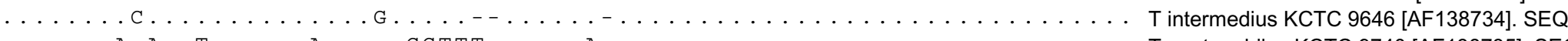

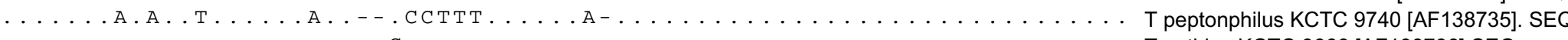
$\ldots \ldots \ldots \ldots \ldots \ldots \ldots \ldots \ldots \ldots \ldots \ldots \ldots \ldots \ldots \ldots \ldots \ldots \ldots \ldots \ldots$ T putidus KCTC 3666 [AF138736].SEQ

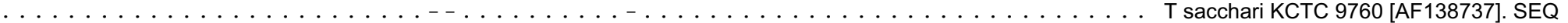
$\ldots \ldots \ldots \ldots \ldots \ldots \ldots \ldots \ldots \ldots \ldots \ldots \ldots \ldots \ldots \ldots \ldots \ldots$ T thalpophilus KCTC 9789 [AF138738]. SEQ

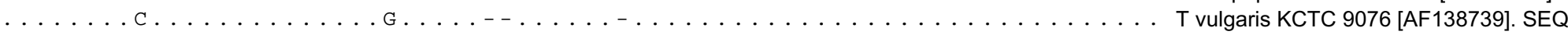

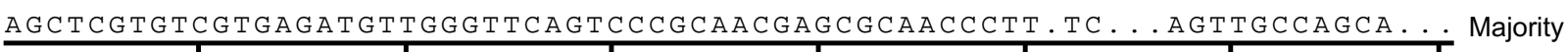
1060 1070 1080 1090 1100 1110
1120

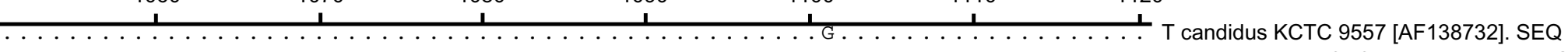

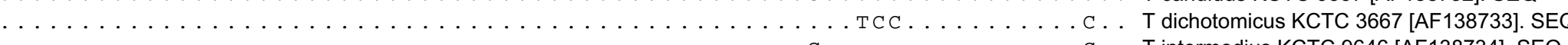

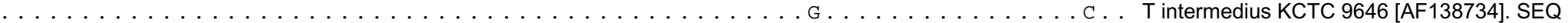

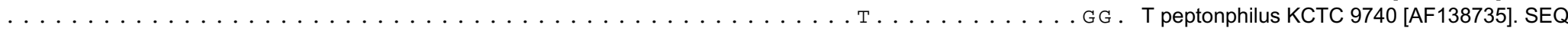

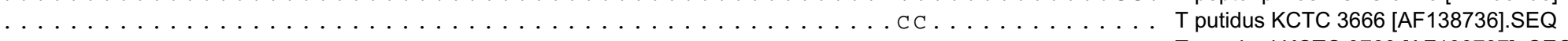

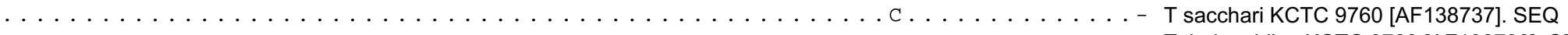
$\ldots \ldots \ldots \ldots \ldots \ldots \ldots \ldots \ldots \ldots \ldots \ldots \ldots \ldots \ldots \ldots \ldots \ldots \ldots \ldots \ldots \ldots$. . . . T thalpophilus KCTC 9789 [AF138738]. SEQ

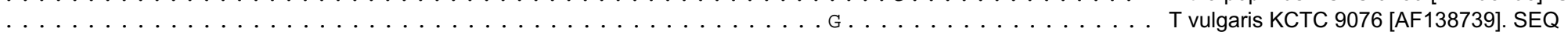

Fig. 1. (continued) 
...G.TGGGCACTCT....GAGACAGCCG. TGAAAG. CGGAGGAAGGTGGGGATGACGTCAAATCATCATG Majority

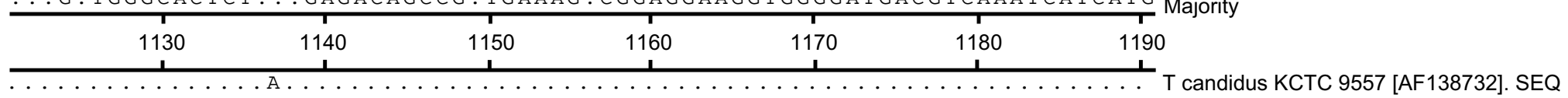

1117

\section{(186}

1186

1158

1186

1143

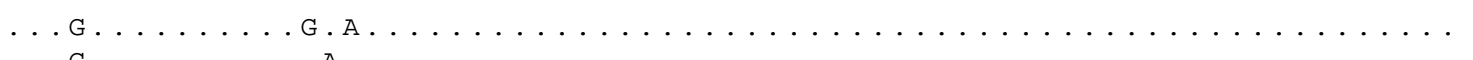

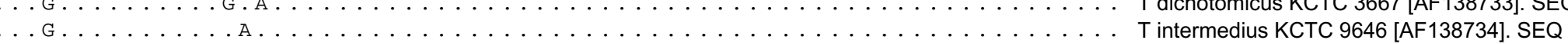

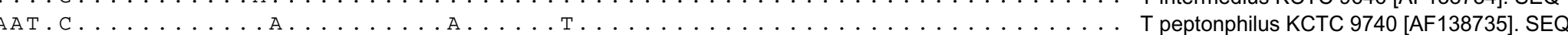

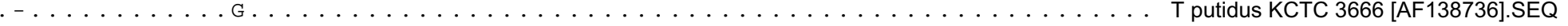

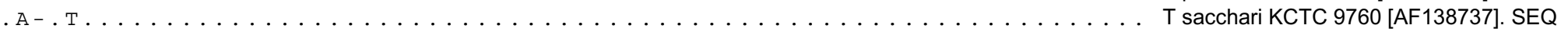

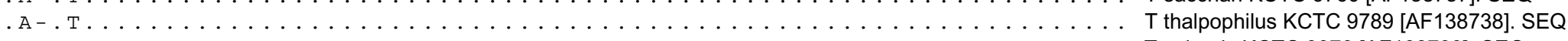

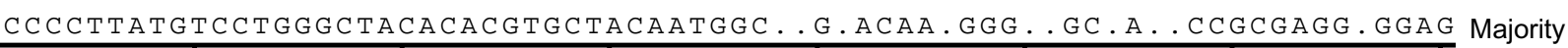

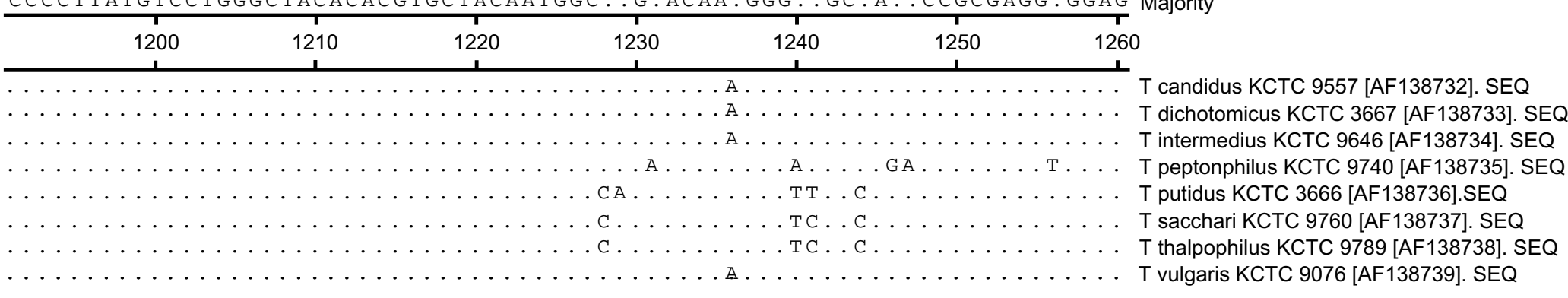
C.AATCCCA.AAA.C..GTCTCAGTTCGGAT.GCAGGCTGCAACTCGCCTGC.TGAAg..GGAATCGCTA Majority

$\begin{array}{rrrrrr}1270 & 1280 & 1290 & 1300 & 1310 & 1320\end{array}$

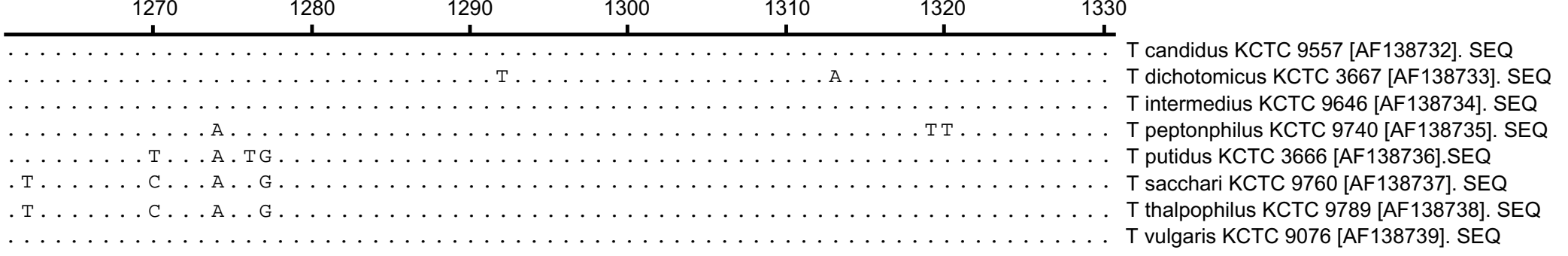

GTAATCGCGGATCAGCATGCCGCGGTGAtACGTtCCCGGGCTtGTACACACCGCCCGTCACACCACG. Majority

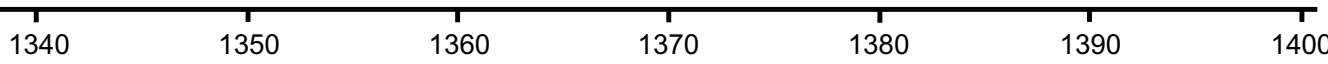

1326

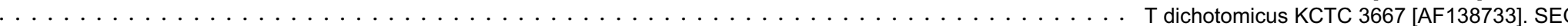

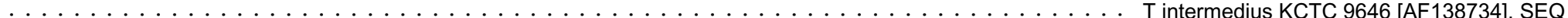
$\ldots \ldots \ldots \ldots \ldots \ldots \ldots \ldots \ldots \ldots \ldots \ldots \ldots \ldots \ldots \ldots \ldots \ldots \ldots \ldots \ldots \ldots \ldots \ldots \ldots \ldots$ T peptonphilus KCTC 9740 [AF138735]. SEQ

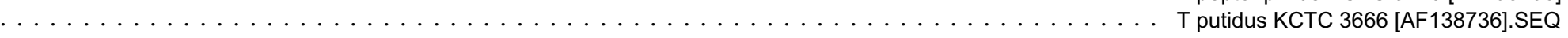

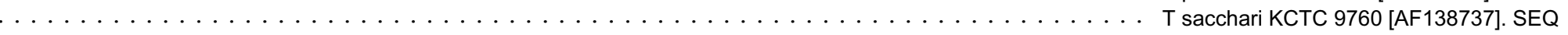
$\ldots \ldots \ldots \ldots \ldots \ldots \ldots \ldots \ldots \ldots \ldots \ldots \ldots \ldots \ldots \ldots \ldots \ldots \ldots \ldots \ldots \ldots$ T thalpophilus KCTC 9789 [AF138738]. SEQ

Fig. 1. (continued) 


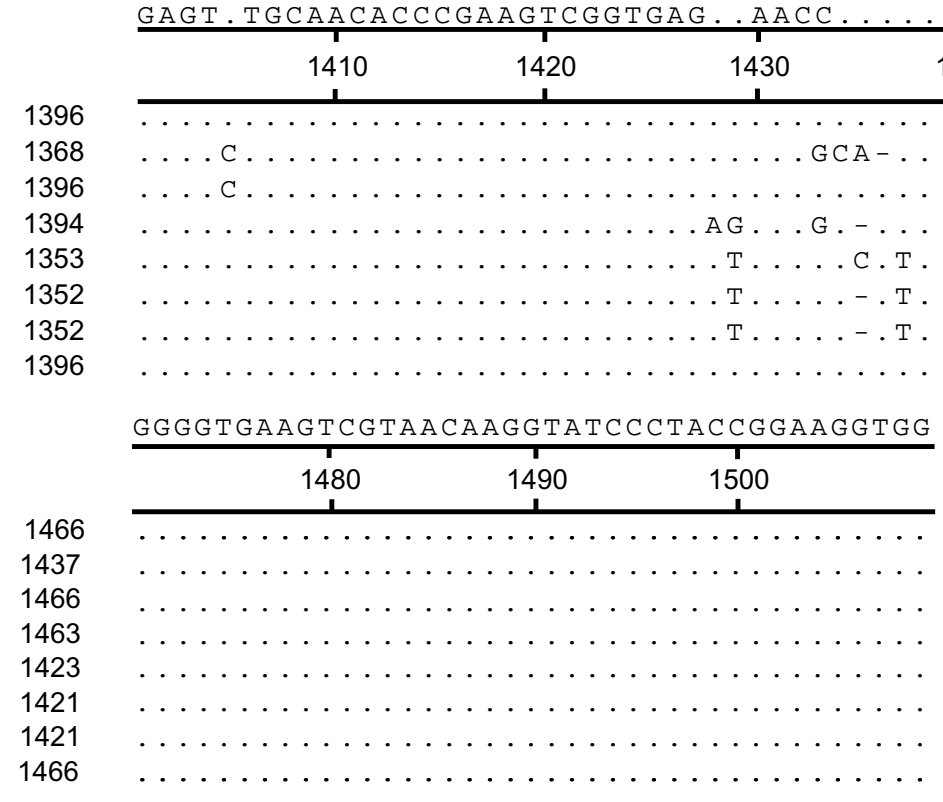

Fig. 1. (continued) 


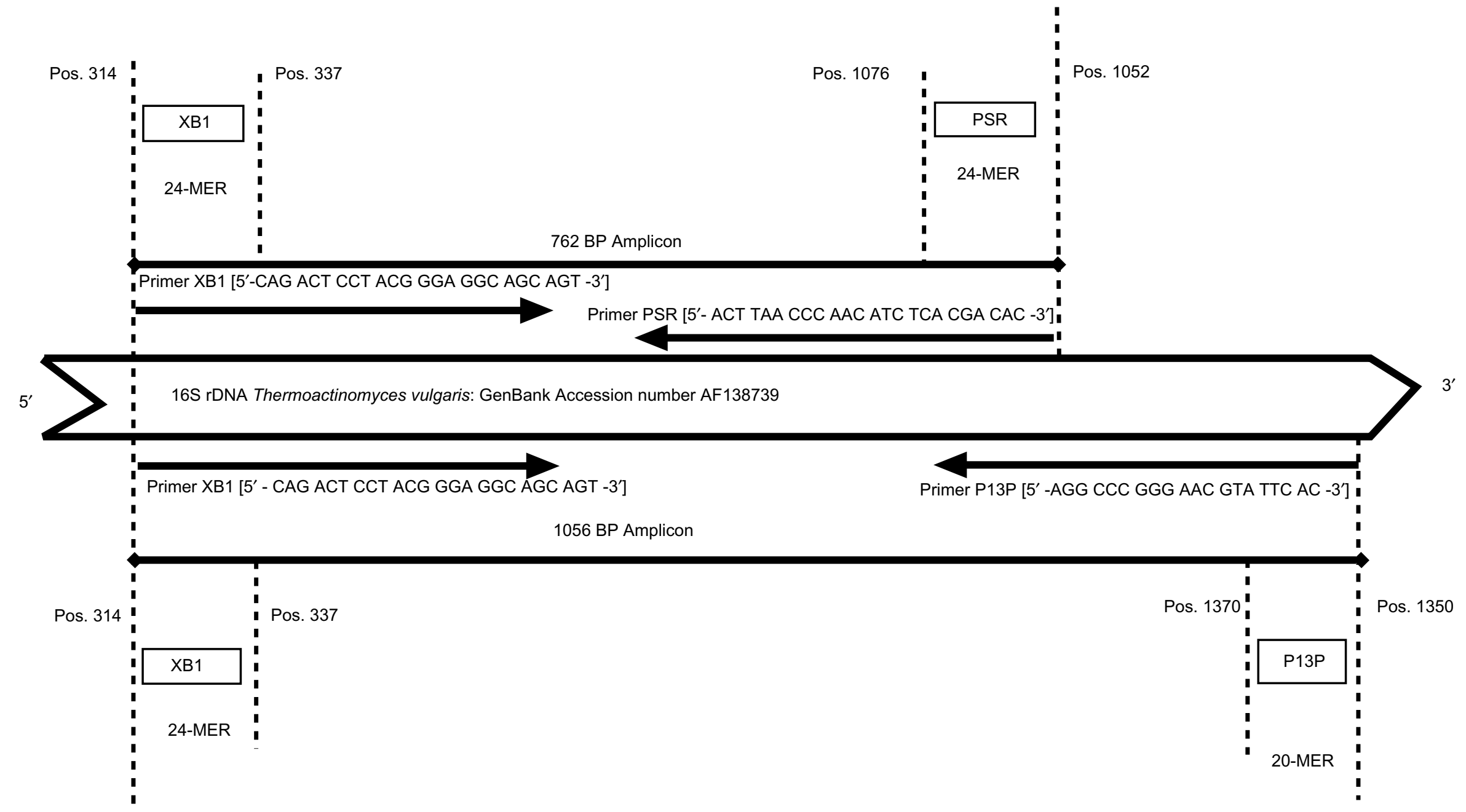

Fig. 2. Primer location, amplicon size and nucleotide sequence of the XB1/PSR and XB1/P13P primer combinations used. 

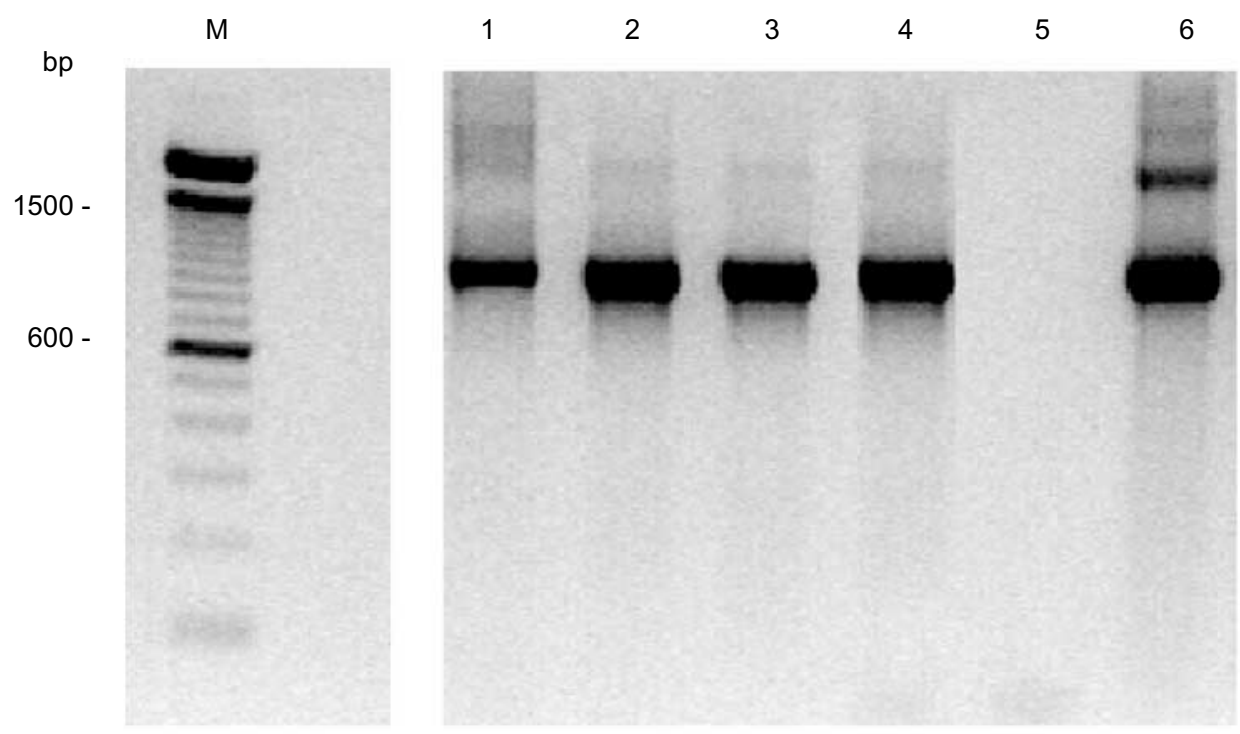

Fig. 3. PCR amplification of a partial $16 \mathrm{~S}$ rDNA region (1056 bp) of four unidentified thermophilic organisms associated with mushroom worker's lung. Lanes 1-6, XB1/P13P primer combination; M, 100-bp mol. wt marker (Life Technologies, Paisley, Scotland). Lane 1, RVH210302; 2, RVH210302B; 3, RVH210302C; 4, RVH210302A; 5, negative control; 6, positive PCR control (S. aureus).

Table 1. Identification of four unidentified thermophilic isolates associated with mushroom worker's lung by PCR amplification and direct sequencing of partial regions of $16 \mathrm{~S}$ rDNA

\begin{tabular}{lclcc}
\hline $\begin{array}{l}\text { Isolate } \\
\text { identifier }\end{array}$ & $\begin{array}{c}\text { Number of } \\
\text { bases } \\
\text { called }\end{array}$ & Identification & $\begin{array}{c}\text { Percentage } \\
\text { homology }\end{array}$ & $\begin{array}{c}\text { Submitted } \\
\text { GenBank } \\
\text { accession no. }\end{array}$ \\
\hline RVH210302 & 1015 & $\begin{array}{l}\text { T. vulgaris } \\
\text { T. candidus }\end{array}$ & 100 & AY114167 \\
RVH210302B & 985 & $\begin{array}{l}\text { T. sacchari } \\
\text { T. thalpophilus }\end{array}$ & 100 & AY114169 \\
RVH210302C & 987 & $\begin{array}{l}\text { T. sacchari } \\
\text { T. thalpophilus }\end{array}$ & 100 & AY114169 \\
RVH210302A & 1001 & Saccharomonospora viridis & 100 & AY114168 \\
\hline
\end{tabular}

before direct DNA sequencing of the $16 \mathrm{~S}$ rDNA amplicon.

Several universal 16S rDNA primers have been described previously including PSR [5] and P13P [6]. The sequence regions they detect were also highly conserved within Thermoactinomyces spp. and hence were suitable for PCR detection of this genus. Examination of the 16S rRNA gene sequence between the eight species demonstrated several hypervariable and conserved regions (Fig. 1). In particular, four hypervariable regions, at positions 400-450, 810-840, $1100-1150$ and 1220-1250, where base diversity in the 16S rRNA gene was highest, were noted. To exploit this heterogeneity for identification purposes, a conserved primer site was sought upstream of position 400. The primer XB1, a 24-mer oligonucleotide, was identified at position 314-337, with reference to $T$. vulgaris AF138739, which was totally conserved within the other seven Thermoactinomyces spp. This primer was combined separately with the reverse primers PSR and P13P, as shown in Fig. 3, with the successful generation of PCR amplicons of the expected sizes. Identification of the MWL isolates was subsequently achieved by direct sequencing of the PCR amplicons with the forward primer (XB1) and gave the results shown in Table 1. BLAST alignment was unable to separate $T$. candidus from $T$. vulgaris, or $T$. thalpophilus from $T$. sacchari. However, recent studies by Yoon et al. [4] based on DNA-DNA hybridisation and 16S rDNA homology have demonstrated that $T$. candidus is a synonym of $T$. vulgaris and T. thalpophilus is a synonym of T. sacchari (Fig. 4).

Overall, the use of the XB1/P13P primer combination is recommended, as this will generate a longer PCR amplicon than that generated by the XB1/PSR primer combination and, more importantly, contains a further two hypervariable regions at base positions 1100-1150 and 1220-1250. In conclusion, this study has identified $16 \mathrm{~S}$ rDNA PCR primer pairs that may be useful in the identification of Thermoactinomyces spp. associated with allergic alveolitis or pneumonitis. 


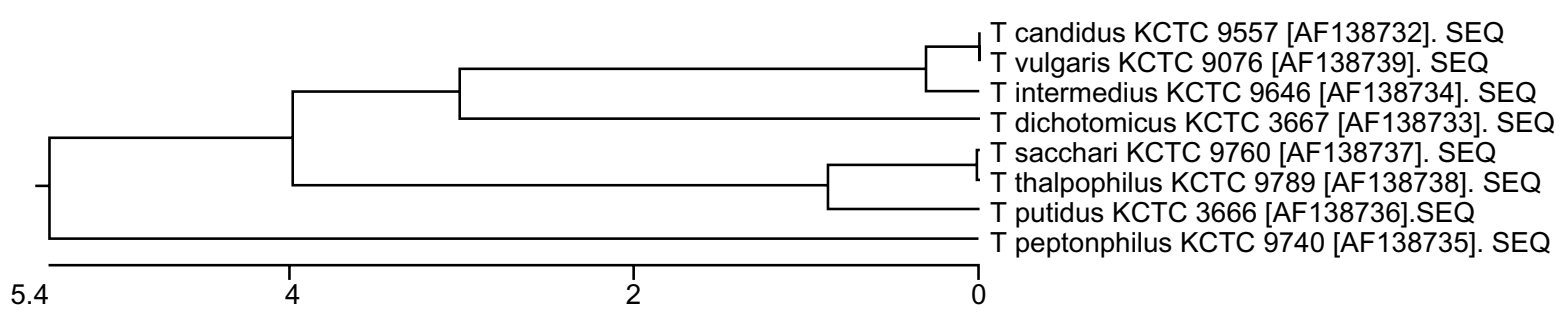

16S rRNA dissimilarity (\%)

Fig. 4. Phylogenetic relationship of the eight described Thermoactinomyces spp. based on complete 16S rRNA gene sequences.

\section{References}

1. Van den Bogart HG, Van den Ende G, Van Loon PC, Van Griensven LJ. Mushroom worker's lung: serologic reactions to thermophilic actinomycetes present in the air of compost tunnels. Mycopathologia 1993; 122: 21-80.

2. Shen YE, Kurup VP, Fink JN. Circulating antibodies against thermophilic actinomycetes in farmers and mushroom workers. J Hyg Epidemiol Microbiol Immunol 1991; 35: 309-316.

3. Kleyn JG, Johnson WM, Wetzler TF. Microbial aerosols and actinomycetes in etiological considerations of mushroom workers' lungs. Appl Environ Microbiol 1981; 41: 1454-1460.

4. Yoon JH, Shin YK, Park YH. DNA-DNA relatedness among Thermoactinomyces species: Thermoactinomyces candidus as a synonym of Thermoactinomyces vulgaris and Thermoactino- myces thalpophilus as a synonym of Thermoactinomyces sacchari. Int J Syst Evol Microbiol 2000; 50: 1905-1908.

5. Campbell PW, Phillips JA, Heidecker GJ, Krishnamani MR, Zahorchak R, Stull TL. Detection of Pseudomonas (Burkholderia) cepacia using PCR. Pediatr Pulmonol 1995; 20: 44-49.

6. Millar B, Moore J, Mallon P et al. Molecular diagnosis of infective endocarditis - a new Duke's criterion. Scand J Infect Dis 2001; 33: 673-680.

7. Millar BC, Xu J, Moore JE Risk assessment models and contamination management: implications for broad-range ribosomal DNA PCR as a diagnostic tool in medical bacteriology. $J$ Clin Microbiol 2002; 40: 1575-1580.

8. Goldenberger D, Kunzli A, Vogt P, Zbinden R, Altwegg M. Molecular diagnosis of bacterial endocarditis by broad-range PCR amplification and direct sequencing. J Clin Microbiol 1997; 35: 2733-2739. 